\title{
R7 Photoreceptor Axon Growth Is Temporally Controlled by the Transcription Factor Ttk69, Which Inhibits Growth in Part by Promoting Transforming Growth Factor- $\beta$ /Activin Signaling
}

\author{
Jonathan S. Kniss, Scott Holbrook, and Tory G. Herman \\ Institute of Molecular Biology, University of Oregon, Eugene, Oregon 97403
}

\begin{abstract}
Work on axon growth has classically focused on understanding how extrinsic cues control growth cone dynamics independent of the cell body. However, more recently, neuron-intrinsic transcription factors have been shown to influence both normal and regenerative axon growth, suggesting that understanding their mechanism of action is of clinical importance. We are studying axon targeting in the Drosophila visual system and here show that the BTB/POZ zinc-finger transcription factor Tramtrack69 (Ttk69) plays an instructive role in inhibiting the growth of R7 photoreceptor axon terminals. Although $t$ tk 69 mutant R7 axons project to the correct medullar target layer, M6, their terminals fail to remain retinotopically restricted and instead grow laterally within M6. This overgrowth is not caused by an inability to be repelled by neighboring R7 axons or by an inability to recognize and initiate synapse formation with postsynaptic targets. The overgrowth is progressive and occurs even if contact between $t$ tk69 mutant R7 axons and their normal target layer is disrupted. Ttk69 is first expressed in wild-type R7s after their axons have reached the medulla; $t$ tk 69 mutant R7 axon terminal overgrowth begins shortly after this time point. We find that expressing Ttk69 prematurely in R7s collapses their growth cones and disrupts axon extension, indicating that Ttk69 plays an instructive role in this process. A TGF- $\beta$ /Activin pathway was shown previously to inhibit R7 axon terminal growth. We find that Ttk69 is required for normal activation of this pathway but that Ttk69 likely also inhibits R7 axon growth by a
\end{abstract} TGF- $\beta$ /Activin-independent mechanism.

\section{Introduction}

The growth cone, a specialized structure at the axon tip, advances, turns, or halts by regulating cytoskeletal actin and microtubules (Vitriol and Zheng, 2012). Growth cones are acutely responsive to their environment and can interpret extrinsic guidance cues without instruction from the cell body. In addition to the four major families of classical guidance cues, many other extrinsic factors, including members of the TGF- $\beta$ family, can influence axon growth (Kolodkin and Tessier-Lavigne, 2011). Past efforts to promote axon regeneration in the CNS have therefore focused on understanding the extrinsic inhibitory signals that prevent injured axons from regrowing. However, recent work has shown that neuron-intrinsic transcription factors also contribute to the varying abilities of axons to grow both during normal development and after injury (Sun and He, 2010; Moore

Received April 25, 2012; revised 0ct. 26, 2012; accepted Nov. 26, 2012.

Author contributions: J.S.K., S.H., and T.G.H. designed research; J.S.K. and S.H. performed research; J.S.K. analyzed data; J.S.K. and T.G.H. wrote the paper.

This work was supported by National Institutes of Health (NIH) National Research Service Award Training Grant 5-T32-HD07348 (J.S.K., S.H.), a Burroughs-Wellcome Career Development Award (T.G.H.), and NIH Grant R01 EY019694 (T.G.H.). We thank C. Doe and A. Travers for antibodies and Z.-C. Lai, L. Zipursky, I. Salecker, C.-H. Lee, the Bloomington Drosophila Stock Center, and the Vienna Drosophila RNAi Center for fly stocks.

Correspondence should be addressed to Dr. Tory Herman, Institute of Molecular Biology, University of Oregon, Eugene, 0R 97403. E-mail: herman@molbio.uoregon.edu.

DOI:10.1523/JNEUROSCI.2023-12.2013

Copyright $\odot 2013$ the authors $\quad 0270-6474 / 13 / 331509-12 \$ 15.00 / 0$ and Goldberg, 2011). Manipulation of these factors can promote axon growth even in the presence of environmental inhibitors, highlighting the potential clinical importance of understanding their properties.

We are studying axon targeting using the R7 photoreceptor neurons in the Drosophila visual system. Each wild-type retina contains $\sim 750 \mathrm{R} 7 \mathrm{~s}$, each of which is paired with an R8 photoreceptor neuron (Wolff and Ready, 1993). R7 axons extend to their final target layer in the medulla, M6, in two main steps (Astigarraga et al., 2010; Hadjieconomou et al., 2011). First, each R7 axon extends along the previously extended $\mathrm{R} 8$ axon to reach the medulla and pauses at a temporary target layer located just beyond the already paused R8 axon terminal. Next, the R7 axon terminals move slowly forward - whether by active migration or because of passive displacement by intercalating neuronal processes remains unclear-until ultimately they form a non-overlapping, retinotopic array in the M6 layer. Previous work has shown that components of a TGF- $\beta$ /Activin signaling pathway, including the Activin receptor Baboon (Babo), are required to prevent overgrowth of R7 axon terminals during the second phase of targeting (Ting et al., 2007): babo mutant R7 axon terminals correctly reach their final M6 target layer but extend laterally within M6, resulting in overlap with adjacent wild-type R7 terminals.

Here we show that the transcription factor Tramtrack69 (Ttk69) plays an instructive role in inhibiting R7 axon growth. Like loss of Babo, loss of Ttk69 causes R7 axon terminals to ex- 
tend laterally within M6. We show that Ttk69 is not required for R7 axons to repel one other or to recognize their synaptic targets, suggesting that Ttk69 instead controls the intrinsic ability of R7 axon terminals to grow. The timing of Ttk69 expression is critical: Ttk69 is specifically expressed in R7s only after their axons have paused in the medulla, ttk69 mutant R7 terminal overgrowth begins shortly afterward, and premature Ttk69 expression causes R7 growth cone collapse. Although we find that Ttk69 is required for normal Activin signal transduction in R7s, we provide evidence that Ttk69 also acts independently of Activin. Together, our results suggest that R7 axon growth is controlled by multiple pathways whose effects are temporally coordinated by expression of a transcription factor.

\section{Materials and Methods}

Genetics. Homozygous wild-type (FRT82), ttk, importin- $\alpha 3$ (imp- $\alpha 3$ ), and syd-1 mutant R7s were generated and labeled using GMR-FLP and MARCM (mosaic analysis with a repressible cell marker) (Lee and Luo, 1999; Lee et al., 2001). When two mutations are on different chromosomes, generating double-mutant R7s using GMR-FLP/MARCM is difficult because of the large number of transgenes that must be present, some on specific chromosome arms. For that reason, we chose to use a mutation in syd-1 (on chromosome 3) and not mutations in Ncad, LAR, or Liprin- $\alpha$ (all on chromosome 2) to disrupt contact between $t$ tk69 mutant R7 axons and their target layer. However, because $s y d-1$ is $<100 \mathrm{~kb}$ away from $t t k$ (http://flybase.org; McQuilton et al., 2012), we chose not to create a syd-1, ttk69 double-mutant chromosome but instead disrupted Ttk69 in syd-1 mutant R7s by creating an imp- $\alpha 3$, syd-1 double-mutant chromosome. Homozygous wild-type (FRT42) and babo mutant R7s were generated and labeled using ey3.5-FLP (from Iris Salecker, MRC National Institute for Medical Research, London, UK) and MARCM. In all MARCM experiments, homozygous cells expressed either UAS- $m C D 8-G F P$ or UASSynaptotagmin $(S y t)-G F P$, along with any other UAS transgenes present, under the control of actin (act)-Gal4. Flies containing the UAS-GFP constructs and act-Gal4 were obtained from the Bloomington Drosophila Stock Center. Because GMR-FLP/MARCM induces mitotic recombination during the final cell division that generates R7s, homozygous R7s inherit Gal80 protein from their heterozygous parents, causing a delay in their expression of transgenes under control of Gal4/UAS (Miller et al., 2009).

In addition to the transgenes used in the MARCM experiments (mentioned above), we also used the following: UAS-ttk69 and UAS-ttk88 (both from the Bloomington Drosophila Stock Center), UAS-ttkRNAi (\#101980), UAS-Ncad-RNAi (\#1093), UAS-babo-RNAi (\#106092), UAS-phyllopod (phyl)-RNAi (\#35469), and UAS-seven in absentia (sina)-RNAi (\#100691) (all from the Vienna Drosophila RNAi Center), UAS-Dcr-2 (Dietzl et al., 2007), UAS-FLAG-dSmad2 (Ting et al., 2007), chaoptin (chp)-Gal4 (a generous gift from S. L. Zipursky, University of California, Los Angeles, CA), and UAS-Gl ${ }^{D N}$ (Allen et al., 1999). We used the following mutations: $t t k^{1 e 11}$ (which we refer to as "ttk69"; Xiong and Montell, 1993), imp- $\alpha 3^{D 93}$ (Mason et al., 2003), babo ${ }^{9}$ (Zheng et al., 2003), syd-1 $1^{w 46}$ (Holbrook et al., 2012), sev ${ }^{V 1}$ (Mullins and Rubin, 1991), and deficiencies spanning the right arm of chromosome 3 (Bloomington Drosophila Stock Center). All animals were raised at $25^{\circ} \mathrm{C}$ except for those analyzed in Figure 7, which were raised at $29^{\circ} \mathrm{C}$. The $U A S-D c r-2$ transgene was included in all RNAi experiments except those in Figure $6 A-C$; we found that the $U A S-t t k-R N A i$ transgene fully eliminates detectable Ttk69 expression even in the absence of UAS-Dcr-2. All sevenless (sev) mutant animals analyzed were male, UAS-ttk88 and UAS-FLAG-dSmad2 experiments were performed with females, and all other experiments were performed with animals of either sex.

Image acquisition and analysis. Brains and retinas were dissected, fixed, and stained by standard methods (Holbrook et al., 2012). Confocal images were collected on a Leica SP2 microscope and analyzed with Leica or Fiji software (http://fiji.sc/Fiji; Schindelin et al., 2012). All quantifications were performed blind.
To quantify nuclear FLAG-dSmad2 levels, we determined the antiFLAG fluorescence intensities within all GFP-labeled R7 nuclei in a field of view (typically two to four) and divided each by the fluorescence intensity within a single non-GFP-expressing (and therefore non-FLAGdSmad2-expressing) R7 nucleus in the same field to calculate a "fold enriched." Anti-Elav staining was used to identify the boundaries of nuclei. To quantify Ttk69 levels in R7s, we determined the average antiTtk69 fluorescence intensity within at least $10 \mathrm{R} 7 \mathrm{~s}$ per retina and corrected for slight differences in gain settings between samples by calibrating the gain using the same fluorophore and otherwise consistent detector settings.

We used the following antibodies: mouse anti-Chp $(24 \mathrm{~B} 10 ; 1: 200)$ and rat anti-Elav (7E8A10; 1:5) from the Developmental Studies Hybridoma Bank; mouse anti-FLAG (M5; 1:200) from Sigma-Aldrich; mouse antiPros (mR1A; 1:500) from C. Doe (University of Oregon, Eugene, OR); rabbit anti-Ttk69 (1:500) from A. Travers (Medical Research Council Laboratory of Molecular Biology, Cambridge, UK); chicken anti-GFP (1:5000) from Abcam; and rabbit anti-GFP (1:5000), Alexa Fluor 555conjugated Phalloidin (1:10) and all secondary antibodies (goat IgG coupled to Alexa Fluor 488, Alexa Fluor 555, or Alexa Fluor 633; 1:250) from Invitrogen.

\section{Results}

Ttk69 is required to prevent overgrowth of $\mathrm{R} 7$ axon terminals To identify genes that regulate R7 axon growth and targeting, we performed a genetic screen using mosaic animals whose R7 neurons were homozygous for randomly mutagenized chromosome arms. We identified three lethal, noncomplementing mutations on $3 \mathrm{R}$ that caused $\mathrm{R} 7$ terminals to overlap with their neighbors. We mapped these by performing complementation tests with a standard set of deficiencies (Bloomington Drosophila Stock Center) and ultimately found that they failed to complement loss-offunction mutations in the $t t k$ gene for lethality. We therefore examined whether loss of $t$ tk caused an R7 axon defect. By $60 \mathrm{~h}$ after puparium formation (APF), wild-type R7 axons terminate in non-overlapping, approximately spherical boutons in the M6 layer of the medulla (Fig. 1A). In contrast, $20.8 \pm 0.70 \%(n=9$ brains) of $t t k^{1 e 11}$ mutant R7 axon terminals extended laterally within the M6 layer, resulting in overlap with adjacent wild-type $\mathrm{R} 7$ terminals (Fig. $1 B, E$ ). Some $t t k^{1 e 11}$ mutant R7s extended beyond their immediate R7 neighbors while remaining in M6 (data not shown), whereas others extended back toward the M3 layer along an adjacent $\mathrm{R} 7$ axon (Fig. $1 B$ ). $t t k^{1 e 11}$ mutant $\mathrm{R} 7$ axons also occasionally extended short branches at the M1 or M3 layers (Fig. $1 C)$. We noticed that even those $t t k^{1 e 11} \mathrm{R} 7$ axon terminals that remained spherical had abnormally large terminals (Fig. 1C,F). We conclude that $t t k$ is required to prevent overgrowth of $\mathrm{R} 7$ axon terminals within the medulla.

The $t$ tk locus encodes two transcription factor isoforms, Ttk69 and Ttk88, which share a common BTB/POZ domain but have different zinc fingers and consequently different DNA-binding specificities (Read and Manley, 1992). The $t t k^{1 e 11}$ allele contains a deletion within a Ttk69-specific exon, completely eliminating Ttk69 expression but leaving Ttk88 expression intact (Lai and Li, 1999). To confirm that the Ttk69 isoform is required for proper $\mathrm{R} 7$ axon growth, we tested the ability of each isoform to rescue the $t$ tk mutant R7 axon defect. We found that overexpressing Ttk88 had no effect on $t t k^{1 e 11}$ mutant R7s (Fig. 1E) but that overexpressing Ttk69 prevented $t t k^{1 e 11}$ mutant R7 axon overgrowth (Fig. 1E; Ttk69 overexpression also caused a gain-of-function phenotype, as described below). We conclude that Ttk69 is required to prevent R7 axon overgrowth and will refer to $t t k^{1 e 11}$ as $t t k 69$. 

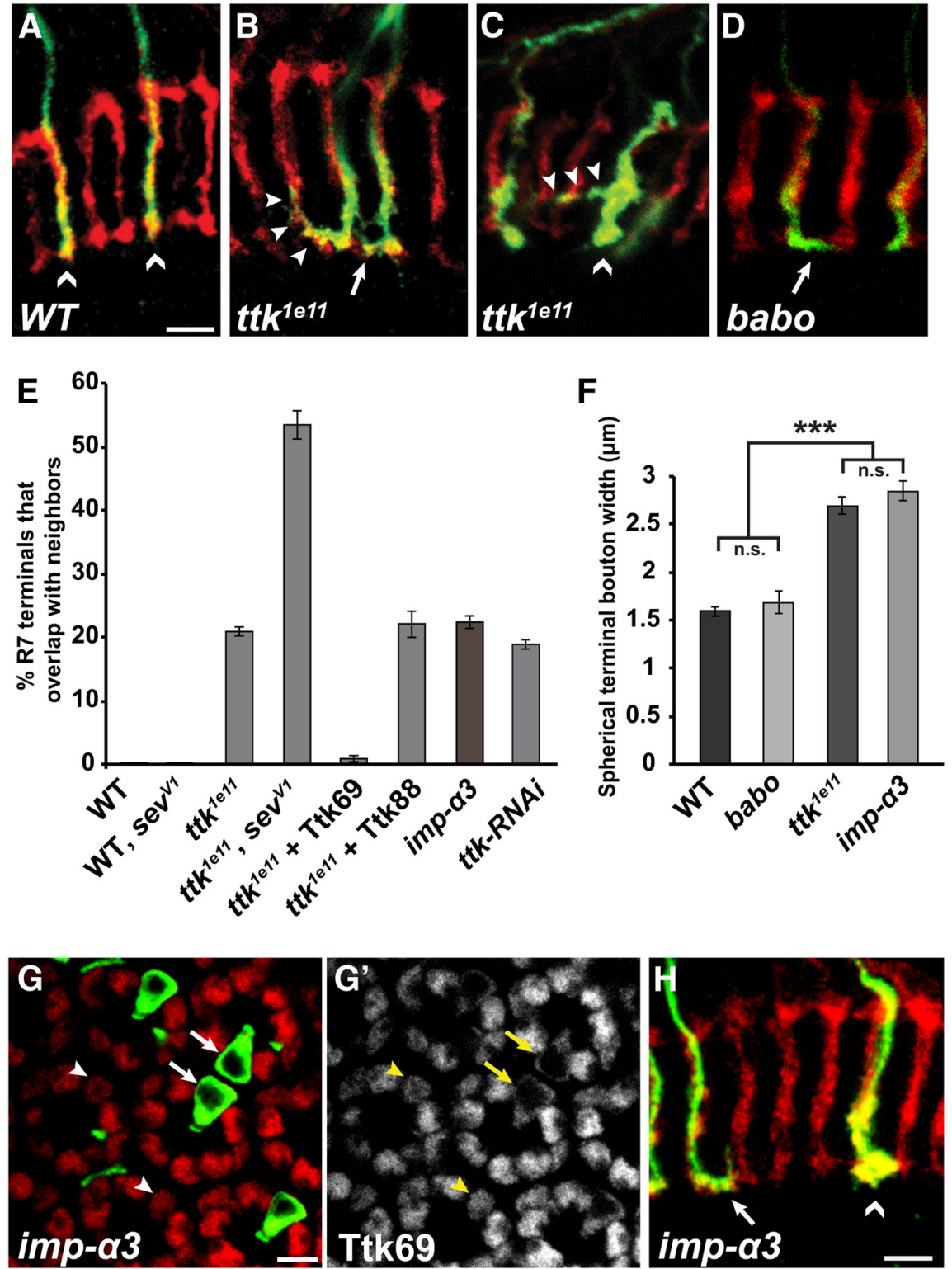

Figure 1. Ttk69 is required to prevent overgrowth of R7 axon terminals. A-C, Medullas of mosaic animals, created by GMR-FLP and MARCM, at $60 \mathrm{~h}$ APF. D, Medulla of a mosaic animal, created by ey3.5-FLP and MARCM, at $60 \mathrm{~h}$ APF. R7s homozygous for the specified chromosome arm express mCD8 -GFP (green). All R7 and R8 axons are labeled with anti-Chp (red). A, Wild-type (FRT82) (WT) R7 axons terminate at the M6 layer of the medulla in spherical boutons (chevrons). $\boldsymbol{B}, t \mathrm{tt}^{1 e 11}$ mutant R7 axons terminate in the M6 layer but often extend laterally (arrows), resulting in overlap with neighboring R7 axon terminals. Some $t t^{1211}$ mutant R7 axons extend not only laterally but also return along an adjacent R7 axon toward the M3 layer (arrowheads). $C$, Even those $t t k^{1 e 11}$ mutant R7 terminals that remain spherical are abnormally large (chevron). $t t k^{1 e 11}$ mutant $R 7$ axons occasionally have lateral branches at the $\mathrm{M} 1$ or $\mathrm{M} 3$ layer $(8.3 \pm 0.7 \% ; n=9$; arrowheads point to an $\mathrm{M} 3$ example). $\boldsymbol{D}$, babo mutant $\mathrm{R} 7$ axons also extend laterally within M6, resulting in overlap with neighboring R7 terminals (arrow), but those babo mutant R7 terminals that remain spherical are of normal diameter. $\boldsymbol{E}$, Quantification of R7 axon extension within M6, scored as the frequency with which individual homozygous R7 axons, created by GMR-FLP/MARCM, overlapped with neighboring R7 axon terminals. If neighboring R7 axons were absent (see below), "overlap" was scored as overlap with the M6 regions directly below neighboring R8 axon terminals in M3 Error bars represent SEM. Zero percent of wild-type (FRT82) R7 axon terminals extend laterally within M6, whether neighboring R7 axons are present (WT; $n=5)$ or have been removed by means of the hypomorphic sev ${ }^{V 1}$ mutation $(n=6)$. The frequency with which $t \mathrm{tk}^{\text {Te11 }}$ mutant R7 axon terminals extend laterally within M6 $(20.8 \pm 0.70 \% ; n=9)$ is enhanced by removing neighboring $\mathrm{R} 7$ axons $(53.3 \pm 2.2 \% ; n=9)$. Expression of the Ttk69 isoform rescues $t \mathrm{tk}^{1 e 11}$ mutant $\mathrm{R} 7$ terminal overlap $(0.8 \pm 0.4 \% ; n=13)$, but expression of Ttk88 does not (21.9 $\pm 2.0 \% ; n=8)$. Individual homozygous wild-type (FRT82) R7 axons expressing ttk-RNAi extend laterally at a frequency $(18.7 \pm 0.77 \% ; n=14)$ similar to that at which $t t^{1 e 11}$ mutant $R 7$ axons do so. The same is true of imp- $\alpha 3$ mutant $\mathrm{R} 7$ axons $(22.3 \pm 1.0 \% ; n=10) . \boldsymbol{F}$, Quantification of the width of $\mathrm{R} 7$ axon terminals that remain spherical. Error bars represent SEM. Both $t t k^{1 e 11}(2.69 \pm 0.10 \mu \mathrm{m}$ in width; $n=9)$ and imp- $\alpha 3(2.84 \pm 0.10 \mu \mathrm{m} ; n=10)$ mutant R7 terminals are significantly larger than either wild-type $(1.58 \pm 0.05 \mu \mathrm{m} ; n=5)$ or babo $(1.67 \pm 0.12 \mu \mathrm{m} ; n=7)$ mutant terminals. ${ }^{* * *} p<0.00001$. There is no significant difference (n.s.) between wild-type and babo R7 terminals ( $\left.p=0.7\right)$ or between $t t k^{1 e 11}$ and imp- $\alpha 3$ terminals $(p=0.2)$. $\mathbf{G}, \boldsymbol{H}$, Mosaic animals created by GMR-FLP/MARCM in which R7s homozygous for imp- $\alpha 3$
Ttk69 is required in the nucleus and specifically affects R7 axon terminal morphology

Ttk69 has been shown previously to act as a transcription factor that represses neuronal development (Read and Manley, 1992; Guo et al., 1995; Giesen et al., 1997; Badenhorst, 2001; Badenhorst et al., 2002). Although some transcription factors have been found to have additional functions outside the nucleus, we did not observe Ttk69 expression in R7 axons (data not shown), and we found that excluding Ttk69 from R7 nuclei by disrupting the nuclear importer Imp- $\alpha 3$ precisely phenocopied loss of Ttk69 (Fig. $1 E-H$ ). We conclude that Ttk69 is likely functioning as a conventional transcription factor to regulate $\mathrm{R} 7$ axon terminals.

Loss of Ttk69 from the entire retina has been shown previously to disrupt adult $\mathrm{R}$ neurons (Lai and Li, 1999). We therefore examined whether the ttk69 mutant R7 axon defect might be secondary to a failure to differentiate properly. By $60 \mathrm{~h} \mathrm{APF}$, wild-type $\mathrm{R} 7$ cell bodies have undergone a change in apical-basal polarity and consequently have a characteristic wedge shape (Fig. 2A; Wolff and Ready, 1993). In addition, they express the neuronal marker Elav (Fig. $2 A^{\prime}$ ), the photoreceptor marker Chp (Fig. 1A), and the R7 fate marker Prospero (Pros; Fig. 2A"), a combination of markers that is unique among retinal cells (Kauffmann et al., 1996). We found that $t$ tk 69 mutant R7 neurons exhibited all the characteristics of normal R7 neurons (Figs. $1 B, 2 B-B^{\prime \prime}$ ) and so conclude that loss of $t$ tk69 does not grossly alter R7 differentiation.

Loss of Ttk69 from non-neuronal cone cells has been shown previously to transform them into R7 neurons (Siddall et al., 2009). Our GMR-FLP/MARCM method of creating ttk69 mutant R7s also creates ttk69 mutant cone cells and, as a consequence, causes some ommatidia to contain extra R7 neurons (Fig. 2B-B"). We found that $49.2 \pm 3.5 \%$ ( $n=7$ retinas) of ommatidia that contained at least one ttk69 mutant R7 (whether the R7 itself or a transformed cone cell) also contained at least one additional R7 neuron. These extra R7s are indistinguishable

express m CD8-GFP (green), $G, G^{\prime}$, At 48 h APF, Ttk69 protein (red) is excluded from the nuclei of imp- $\alpha 3$ mutant R7s (arrows; compare with wild-type R7s, arrowheads). $\boldsymbol{H}$, At $60 \mathrm{~h}$ APF, imp- $\alpha 3$ mutant $R 7$ terminals extend laterally within M6 (arrows). Those imp- $\alpha 3$ mutant R7 terminals that remain spherical are abnormally large (chevron). Scale bars, $5 \mu \mathrm{m} . n$ equals number of brains scored. 
from the native R7s in shape and in their expression of R7 markers (Fig. $2 B-B^{\prime \prime}$ ). However, because we cannot unambiguously trace the origin of each $t$ tk $69 \mathrm{mu}-$ tant R7 axon, we were concerned that these ectopic R7s might somehow be abnormal and exclusively responsible for the observed axon morphology defect. To test this, we needed to remove Ttk69 from R7s without disrupting any nonneuronal cells. To do so, we used the $\mathrm{R}$ neuron-specific driver chp-Gal4 to drive expression of a $t t k-R N A i$ transgene (Fig. $2 C-F^{\prime}$ ). We found that this technique specifically eliminated Ttk69 expression from $\mathrm{R}$ neurons and therefore did not result in the formation of extra R7 neurons (Fig. $2 F, F^{\prime}$ ). However, R7 axon terminals consequently extended laterally within the M6 layer, causing overlap with adjacent R7s at a frequency similar to that observed for individual ttk69 mutant R7 axons $(21.8 \pm 2.6 \% ; n=9$ brains; Fig. $2 E)$. We conclude that Ttk69 is normally required in R7 neurons to prevent abnormal axon terminal growth. In additional support of this conclusion, we found that using GMR-FLP/MARCM to drive expression of the $t t k-R N A i$ transgene in individual homozygous wild-type (FRT82) cells did not cause nonneuronal cells to become extra R7s [data not shown; presumably this is because of the delay in expression (see below and Materials and Methods)] but did cause R7 axon terminals to extend laterally within M6 (18.7 \pm $0.77 \% ; n=14$ brains; Fig. $1 E$ ).

\section{ttk69 mutant $\mathrm{R} 7$ axons are still} responsive to repulsion by their neighbors

Previous studies have shown that wildtype R7 axons will extend laterally within M6 if two conditions are met: (1) there is at least one extra R7 in the same ommatidium, causing multiple R7 axon terminals to occupy the M6 space normally reserved for one (a condition we will refer to as "crowding"); and (2) neighboring R7 axons have been removed (Ashley and Katz, 1994). Under these circumstances, crowded R7 axon terminals will specifically extend into M6 regions lacking R7 innervation, leading to the conclusion that R7 axons are mutually repulsive but that a cell-intrinsic mechanism is normally sufficient to prevent R7 axon overlap (Ashley and Katz, 1994). As mentioned in the previous section, ttk69 mutant R7 axons can be crowded, satisfying condition 1. One possibility, therefore, is that $t t k 69$ mutant R7 axons extend laterally within M6 because they are unable to detect the presence of adjacent R7 axons, satisfying condition 2 . If this were the case, we would predict that removing adjacent $\mathrm{R} 7$ axons would have no effect on the ability of $t$ tk69 mutant terminals to overlap with
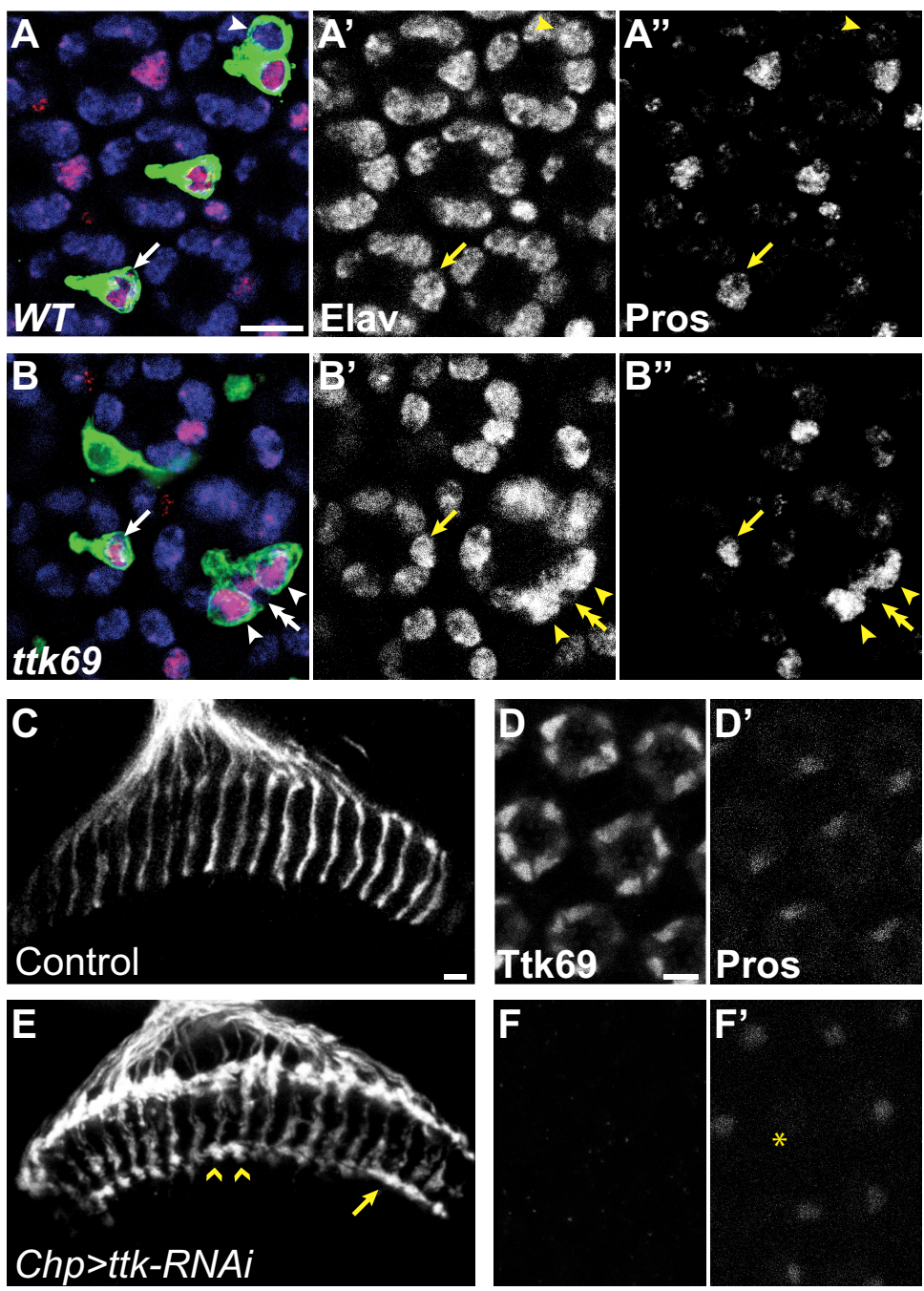

Figure 2. Ttk69 specifically affects R7 axon terminal morphology. $\boldsymbol{A}, \boldsymbol{B}$, Retinas of mosaic animals, created by GMR-FLP/ MARCM, at $60 \mathrm{~h}$ APF. Homozygous R7s express mCD8 -GFP (green). $A-A^{\prime \prime}$, Wild-type (FRT82) (WT) R7 cell bodies (arrow) express the neuronal marker Elav (blue; $A^{\prime}$ ) and the R7 fate marker Pros (red; $A^{\prime \prime}$ ). The GMR-FLP/MARCM technique also creates homozygous R1 and R6 neurons (arrowhead), which express Elav but not Pros. $\boldsymbol{B}-\boldsymbol{B}^{\prime \prime}$, Native R7s that are homozygous for $t$ tk69 (ttk ${ }^{1 e 11}$; arrow) correctly express Elav $\left(\boldsymbol{B}^{\prime}\right)$ and Pros $\left(\boldsymbol{B}^{\prime \prime}\right)$. Cone cells homozygous for ttk69 are transformed into R7s (arrowheads) and also express Elav and Pros. Transformed ttk69 mutant cone cells can be positioned anywhere within the ommatidium. In this example, two transformed cone cells flank the native, wild-type R7 (double arrowhead), displacing the latter into a slightly different focal plane. C, Medulla of a wild-type adult animal in which R1-R8 neurons express EB1-GFP (white) under the control of chp-Gal4. The R7 axons terminate in non-overlapping boutons in M6. $\boldsymbol{D}, \mathbf{D}^{\prime}$, Retina from a wild-type $60 \mathrm{~h}$ APF pupa in which R1-R8 neurons express EB1-GFP under the control of chp-Gal4. All R neurons express Ttk69 (D), and each ommatidium contains exactly one R7 neuron, as judged by Pros expression $\left(\boldsymbol{D}^{\prime}\right)$. $E$, Medulla of a wild-type adult animal in which R1-R8 neurons express both EB1-GFP (white) and ttk-RNAi under the control of chp-Gal4. R7 axons in these animals extend laterally within M6, resulting in overlap (21.8 $\pm 2.6 \% ; n=7$ brains; arrow), or have abnormally large terminals (chevrons). $\boldsymbol{F}, \boldsymbol{F}^{\prime}$, Retina from a $60 \mathrm{~h}$ APF pupa in which R1-R8 neurons express both EB1-GFP and ttk-RNAi under the control of chp-Gal4. Ttk69 protein is absent from all R neurons $(\boldsymbol{F})$ but each ommatidium still contains exactly one R7, as judged by Pros expression $\left(\boldsymbol{F}^{\prime}\right)$; the asterisk indicates an ommatidium in which the Pros-positive R7 is present but out of the plane of focus. Scale bars, $5 \mu \mathrm{m}$.

their neighbors. However, when we used a hypomorphic sev allele to eliminate most R7s, those $t$ tk69 mutant R7 axons that remained were significantly more likely to extend laterally within M6 (Fig. 1E) We conclude that $t$ tk69 mutant R7 axons are still responsive to repulsion by adjacent R7 axons.

\section{ttk69 mutant $\mathrm{R} 7$ axons correctly recognize their synaptic} target layers

In addition to being repelled by their neighbors, R7 axons presumably also receive cues from their synaptic targets. Although disrupting presynaptic assembly within R7s does not cause their 

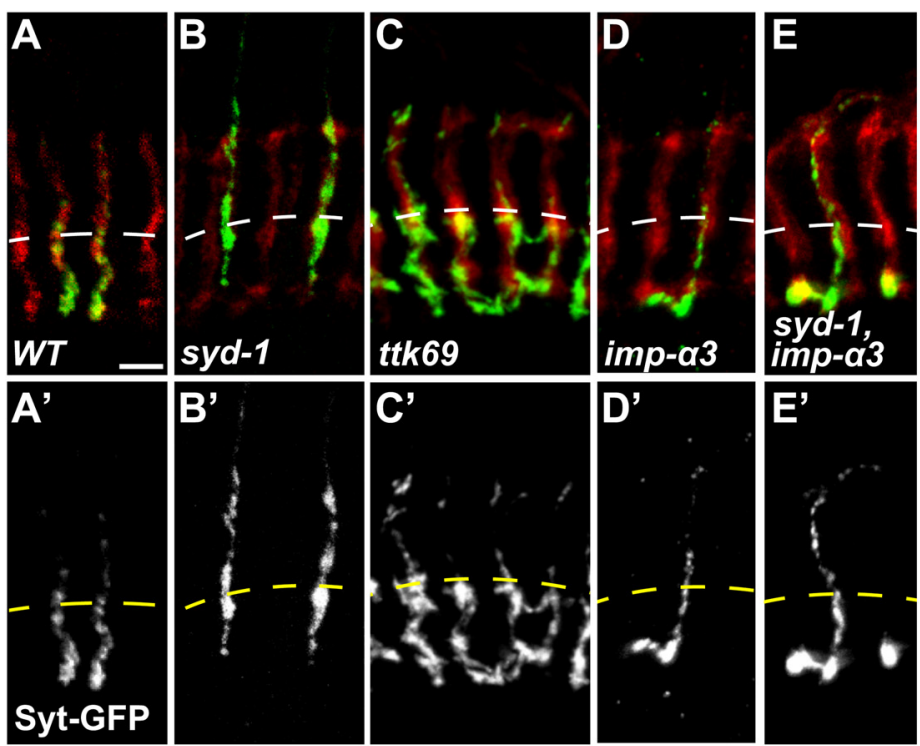

G

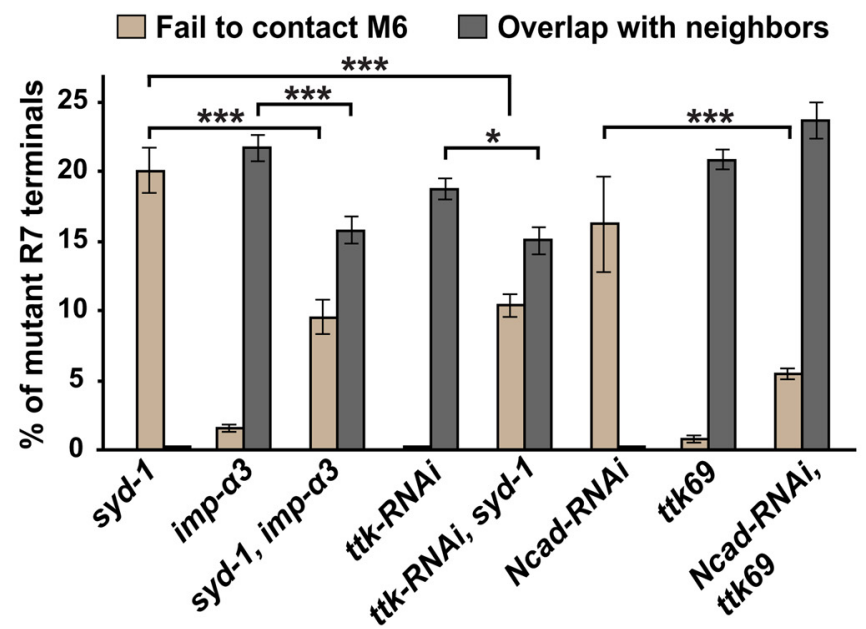

Figure 3. ttk69 mutant R7 axons correctly identify their synaptic target layer yet still overgrow if contact with their target layer is disrupted. $\boldsymbol{A}-\boldsymbol{F}^{\prime}$, Medullas of mosaic animals, created by GMR-FLP/MARCM, at $60 \mathrm{~h}$ APF. R7s homozygous for the specified chromosome arm express Syt-GFP (green). All R7 and R8 axons are labeled with anti-Chp (red). $\boldsymbol{A}, \boldsymbol{A}^{\prime}$, Wild-type (FRT82) (WT) R7s primarily localize Syt-GFP to the region of the axon below the M3 layer (dashed line). $B, B^{\prime}$, syd-1 mutant R7s mislocalize Syt-GFP to regions of the axon that lie within and above the M3 layer. syd-1 mutant R7 axons often fail to contact M6. In $t t k 69\left(t t k^{1 e 11} ; \mathbf{C}, \boldsymbol{C}^{\prime}\right)$ and imp- $\alpha 3\left(\boldsymbol{D}, \boldsymbol{D}^{\prime}\right)$ mutant $\mathrm{R} 7$ axons Syt-GFP is primarily localized below $\mathrm{M} 3$, as in wild type. $\boldsymbol{E}-\boldsymbol{F}^{\prime}$, imp- $\alpha 3$, syd-1 double-mutant R7 axons mislocalize Syt-GFP yet extend laterally within M6.F, Even double-mutant R7s that fail to contact M6 can still extend laterally (arrowheads). G, Quantification of R7 axons that either extend within or fail to contact M6. Error bars represent SEM. LoSs of imp- $\alpha 3$ greatly increases the frequency with which syd-1 mutant R7 axons contact M6 [only $9.5 \pm 1.3 \%(n=19)$ of imp- $\alpha 3$, syd-1 R7 axons fail to contact M6 compared with $20.0 \pm 1.6 \%(n=13)$ of syd-1 mutant axons]. Loss of syd-1 moderately decreases the ability of imp- $\alpha 3$ mutant axons to extend laterally [15.8 $\pm 1.0 \%(n=19)$ of imp- $\alpha 3$, syd-1 R7 axons overlap with neighboring R7 axons compared with $21.6 \pm 0.93 \%(n=11)$ of imp- $\alpha 3$ mutant axons]. Similarly, disrupting ttk with ttk-RNAi greatly increases the frequency with which syd- 1 mutant R7 axons contact M6 [only $10.3 \pm 0.84 \%(n=13)$ of $t$ tk-RNAi-expressing syd- 1 mutant R7 axons fail to contact M6], and loss of syd- 1 moderately decreases the ability of ttk-RNAi-expressing R7s to extend laterally [only $15.0 \pm 0.99 \%(n=13)$ of $t$ tk-RNAi-expressing syd-1 mutant R7 axons extend within M6 (the percentage of $t t k-R N A i$-expressing wild-type $\mathrm{R} 7$ axons that do so is redisplayed from Fig. 1 Efor ease of comparison]. Finally, loss of ttk69 likewise greatly increases the frequency with which Ncad-RNAi-expressing R7 axons contact M6 [only $5.5 \pm 0.44 \%(n=14)$ of NcadRNAi-expressing $t t k 69\left(t t k^{1711}\right)$ mutant R7 axons fail to contact M6 compared with $16.2 \pm 3.4 \%(n=7)$ of Ncad-RNAi-expressing wild-type R7 axons]. Ncad-RNAi does not significantly affect the frequency with which ttk69 mutant R7 axons extend laterally [23.6 $\pm 1.3 \%(n=14)$ of Ncad-RNAi-expressing ttk69 mutant R7 axons overlap with neighboring R7 axons (the percentage of ttk69 mutant $\mathrm{R} 7$ axons that do so is redisplayed from Fig. $1 E$ for ease of comparison); $p=0.12$ ]. Error bars represent $S E M .{ }^{*} p<$ 0.01 and ${ }^{* * *} p<0.0001$. Scale bar, $5 \mu \mathrm{m}$. $n$ indicates number of brains scored.

axons to extend laterally (Clandinin et al., 2001; Lee et al., 2001; Maurel-Zaffran et al., 2001; Choe et al., 2006; Hofmeyer et al., 2006; Astigarraga et al., 2010; Holbrook et al., 2012), it remained possible that the $t t k 69$ mutant $\mathrm{R} 7$ axon defect might nonetheless
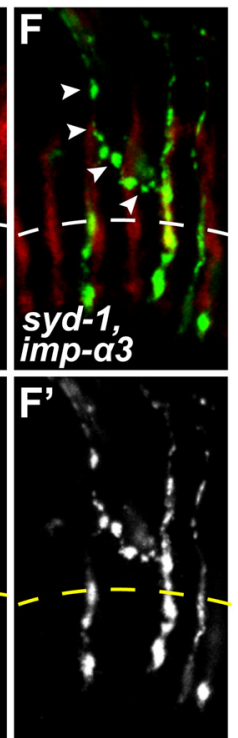

be caused by an inability to recognize synaptic targets or might reflect a failure to transition from axon growth to synaptogenesis. Indeed, although wild-type R7 terminals lack filopodia and are instead smooth, ellipsoid boutons by $60 \mathrm{~h} \mathrm{APF}$ (Fig. 1A), ttk69 mutant R7 terminals failed to form normal boutons and instead were irregular in shape and had short, filopodia-like extensions (Fig. 1B). To distinguish whether $t$ tk69 mutant R7 axons recognize their synaptic targets, we examined their ability to localize the synaptic vesicle marker Syt-GFP. In wild-type R7 axons, Syt-GFP is localized primarily below the M3 layer, consistent with the known locations of presynaptic sites (Fig. $3 A, A^{\prime}$; Takemura et al., 2008). However, R7s lacking the presynaptic assembly proteins Syd-1 or Liprin- $\alpha$ mislocalize SytGFP to puncta along the length of the axon (Fig. 3B, $B^{\prime}$; Holbrook et al., 2012). We found that $t$ tk 69 mutant R7s correctly localized Syt-GFP below the M3 layer (Fig. $3 C, C^{\prime}$ ), although the amount of SytGFP in this region appeared to be increased in ttk69 mutant R7 axons (Fig. $\left.3 C, C^{\prime}\right)$, perhaps because of their expanded size. This result suggests that $t t k 69$ mutant R7s correctly identify their synaptic targets and can at least partly assemble synapses. In addition, $t$ tk69 mutant R7 axons never extended outside the layers within which they normally form synapses: in particular, most remained within M6, and those that instead turned back along adjacent R7 axons never extended beyond M4 (Fig. 1B). This result suggests that the overgrowth of $t$ tk69 mutant R7 terminals is restricted to regions that contain their normal synaptic targets.

ttk69 mutant $\mathrm{R} 7$ axons still overgrow if contact with their M6 target layer is disrupted

The restriction of $t t k 69$ mutant $\mathrm{R} 7$ axon terminals to regions containing R7 synaptic targets suggested that their ability to overgrow might require contact with M6 or the ability to form synapses. To test this, we set out to disrupt the latter processes in R7s lacking Ttk69. Loss of Ncad, LAR, Liprin- $\alpha$, or Syd-1 reduces the size of R7 terminal boutons and causes many to fail to contact M6 (Clandinin et al., 2001; Lee et al., 2001; Maurel-Zaffran et al., 2001; Choe et al., 2006; Hofmeyer et al., 2006; Holbrook et al., 2012). For technical reasons, we were unable to create R7s doubly mutant for $t$ tk 69 and $N c a d$, $L A R$, or Liprin- $\alpha$ (see Materials and Methods). Instead, we took the following three approaches to disrupt both $t t k 69$ and contact with M6: (1) we created imp- $\alpha 3$, syd-1 double-mutant 
R7s [loss of imp- $\alpha 3$ eliminates nuclear Ttk69 expression and exactly phenocopies all aspects of the $t t k 69$ mutant R7 axon defect (Fig. $1 E-H^{\prime}$ ), including normal localization of Syt-GFP (Fig. $\left.3 D, D^{\prime}\right)$ ]; (2) we disrupted Ttk69 protein in syd-1 mutant R7s by causing the latter to express ttk-RNAi; and (3) we disrupted Ncad protein in ttk69 mutant R7s by causing the latter to express $N c a d-R N A i$. We obtained similar results in all three cases. As expected, loss of syd-1 from imp- $\alpha 3$ mutant R7s disrupted their ability to localize Syt-GFP and to maintain contact with M6 (Fig. $3 E-G$ ), as did loss of syd-1 from $t t k-$ $R N A i$-expressing R7s (Fig. $3 G$ ) or causing ttk69 mutant R7s to express Ncad-RNAi (Fig. 3G). However, even those imp- $\alpha 3$, syd-1 double-mutant R7 axons that failed to contact M6 were able to extend laterally (Fig. $3 F, F^{\prime}$ ), suggesting that $t t k 69 \mathrm{mu}-$ tant R7 axon overgrowth does not require contact with synaptic targets. In addition, significantly more imp- $\alpha 3$, syd-1 double-mutant and $t t k-R N A i$-expressing syd-1 mutant R7 axons made contact with M6 than did syd-1 single-mutant R7 axons (Fig. 3G), suggesting that loss of Ttk69 from syd-1 mutant R7s may cause their axons to overgrow not only laterally but also toward M6. Similarly, more Ncad-RNAi-expressing ttk69 mutant R7 axons made contact with M6 than did Ncad$R N A i$-expressing wild-type $\mathrm{R} 7$ axons (Fig. $3 G$ ), again indicating that loss of Ttk69 causes R7 axons lacking Ncad to overgrow toward M6. However, we do note that loss of syd-1 from imp- $\alpha 3$ mutant R7s and loss of syd-1 from ttk-RNAiexpressing R7s reduces the frequency with which their axons extend laterally (Fig. $3 G$ ), indicating that, although not absolutely required, stable contact with M6 may facilitate R7 axon overgrowth.

\section{Ttk69 is first expressed in wild-type R7s after their axons extend into the medulla; $t$ tk69 mutant R7 axon terminal overgrowth begins shortly afterward}

We have so far shown that loss of Ttk69 causes R7 axon terminals to overgrow, that this overgrowth does not require contact with the normal R7 synaptic target layer, and that this overgrowth is not caused by an inability to be repelled by neighboring R7 axons or to recognize the appropriate synaptic targets. Together, these results indicate that Ttk69 is required to downregulate some R7-intrinsic mechanism that promotes axon terminal growth. We next wanted to determine whether Ttk69 might also be sufficient to downregulate R7 axon growth and so play an instructive rather than simply permissive role in this process. To do so, we first determined the time course of Ttk69 expression to compare it with that of wildtype R7 axon outgrowth. Previous work has shown that Ttk69 is absent from cells as they differentiate into R neurons during larval development (Li et al., 1997) but is present at high levels in all $\mathrm{R}$ neurons at $40 \mathrm{~h} \mathrm{APF} \mathrm{(Lai} \mathrm{and} \mathrm{Li}, 1999$ ). If Ttk69 is indeed instructive rather than merely permissive, one prediction is that it should be absent from R7 axons that are actively extending. We therefore expected that R7s would not express Ttk69 as they initially extend to their temporary target layer. Because R7s are specified by a wave of differentiation that passes across the developing retina (Wolff and Ready, 1993), the initial extension of R7 axons occurs sequentially rather than simultaneously and is complete by $\sim 30 \mathrm{~h} \mathrm{APF} \mathrm{(Ting} \mathrm{et}$ al., 2005). Indeed, we found that Ttk69 was still undetectable in R7s at $24 \mathrm{~h} \mathrm{APF}$, when most R7 axons have completed their initial extension into the medulla (Fig. $4 A, A^{\prime}, L$ ). However, by
$30 \mathrm{~h} \mathrm{APF}$, Ttk69 was expressed at high levels in all R7s (Fig. $\left.4 B, B^{\prime}, L\right)$. Ttk69 levels then decreased but persisted throughout the second phase of R7 axon targeting (Fig. $4 C-E^{\prime}, L$ ).

The absence of Ttk69 from R7s whose axons are actively extending is consistent with the possibility that Ttk69 might be an instructive signal for R7s to decrease axon growth. If such a model were true, we would also predict that $t$ tk69 mutant R7 axons should exhibit increased growth shortly after Ttk69 would normally be expressed. Indeed, we found that wild-type and $t$ tk 69 mutant R7 axons were indistinguishable at both $24 \mathrm{~h}$ APF (data not shown) and $30 \mathrm{~h} \mathrm{APF} \mathrm{(Fig.} \mathrm{4F,} \mathrm{I).} \mathrm{However,} \mathrm{by}$ $36 \mathrm{~h} \mathrm{APF}, 10.1 \pm 0.8 \%$ ( $n=10$ brains $)$ of $t$ tk 69 mutant R7 axon terminals overlapped with their neighbors (Fig. $4 G, J, M)$, and by $42 \mathrm{~h} \mathrm{APF}$, this overlap was as frequent as that observed at $60 \mathrm{~h}$ APF (Fig. $4 H, K, M$ ). The average length of ttk69 mutant lateral extensions continued to increase between 48 and $60 \mathrm{~h} \mathrm{APF} \mathrm{(Fig.} \mathrm{4M).} \mathrm{Together,} \mathrm{these} \mathrm{results} \mathrm{are} \mathrm{consis-}$ tent with a model in which the timing of Ttk69 expression coincides with the second phase of R7 targeting, during which Ttk69 inhibits R7 axon growth.

\section{Premature Ttk69 expression disrupts R7 axon extension}

To directly test whether Ttk69 might be sufficient to inhibit R7 axon growth, we wanted to drive expression of Ttk69 prematurely, before the R7 axons have reached their temporary target layer. However, expressing Ttk69 in R7 precursors before they are committed to a neuronal fate has been shown previously to prevent their differentiation as R7s (Li et al., 1997; Siddall et al., 2009). To avoid this outcome, we therefore needed to delay ectopic expression of Ttk69 until after the R7s are specified. We chose not to use a global driver, such as the heat shock promoter, because that would result in Ttk69 induction simultaneously in all R7s despite their varying developmental ages; at any given time point, many would not yet be committed to the neuronal fate. Instead, we took advantage of the same GMR-FLP/MARCM system that we use to generate and label individual homozygous R7s. We have found that GMR-FLP/MARCM causes each homozygous R7 to express UAS-driven transgenes $\sim 10-15 \mathrm{~h}$ after its recruitment as an R7 and causes their axons to be GFP labeled before they have reached their temporary target layer (Ting et al., 2005; Miller et al., 2009). We therefore used GMR-FLP/MARCM to express UAS-ttk69 in wild-type R7s. By $24 \mathrm{~h}$ APF, most wild-type R7 axons have reached their temporary target layer in the medulla, although some have not (Fig. 5A; Ting et al., 2005). Although R7s forced to express Ttk69 prematurely can still reach their temporary target layer, we noticed that some appeared to terminate instead in the R8 temporary target layer and had abnormally thin terminals (Fig. $5 B, B^{\prime}$ ). This result suggested that premature Ttk69 might collapse R7 growth cones and so inhibit axon growth.

To test whether premature Ttk69 actually prevents R7 axons from reaching their targets, we next examined GMR-FLP/ MARCM/UAS-ttk69 R7s at $48 \mathrm{~h}$ APF, by which time all wild-type R7 axons have reached their temporary target layer and have begun their slow advance to their final destination, M6. We found that $17.6 \pm 1.3 \%$ ( $n=9$ brains) of wild-type R7 axons forced to express Ttk69 were absent from their normal target layer and instead terminated in the same layer as R8 axons (Fig. $5 C$ ); some of these apparently stalled axons extended thin 
$24 \mathrm{hr}$ APF
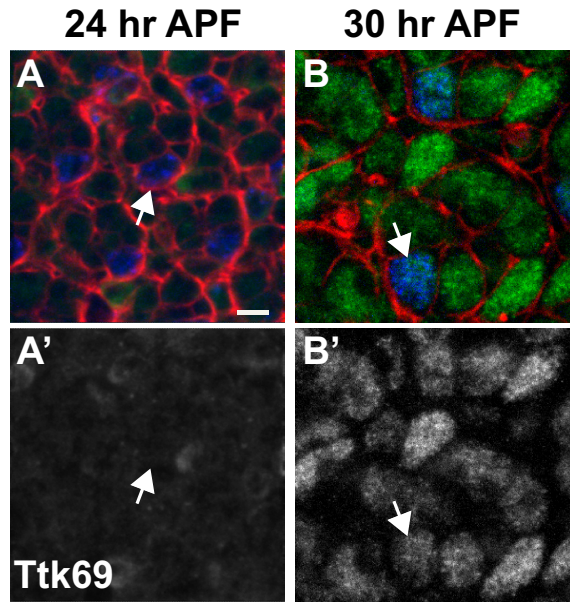

$36 \mathrm{hr}$ APF
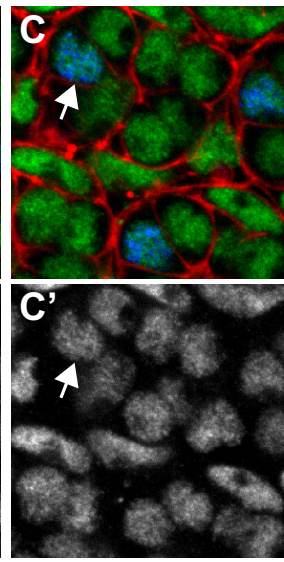

$48 \mathrm{hr}$ APF
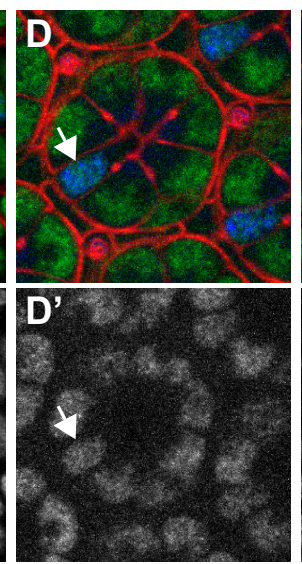

$60 \mathrm{hr}$ APF

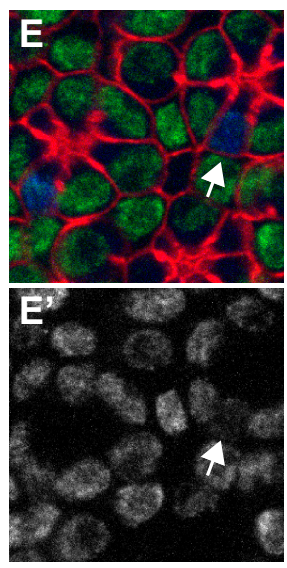

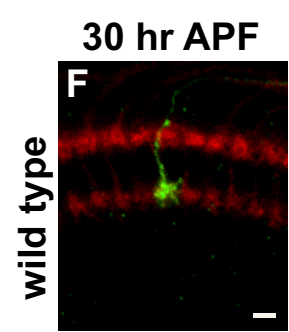

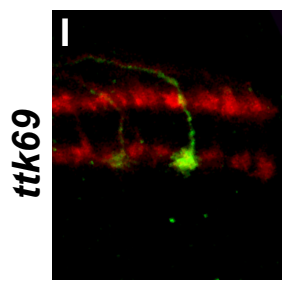

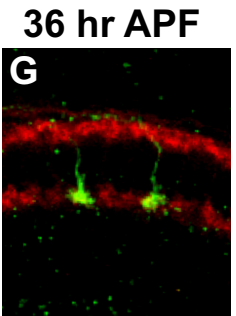

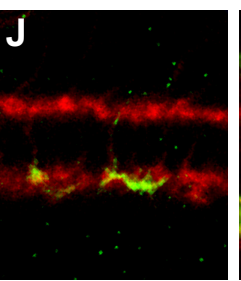

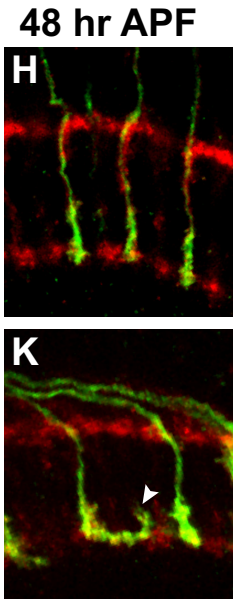
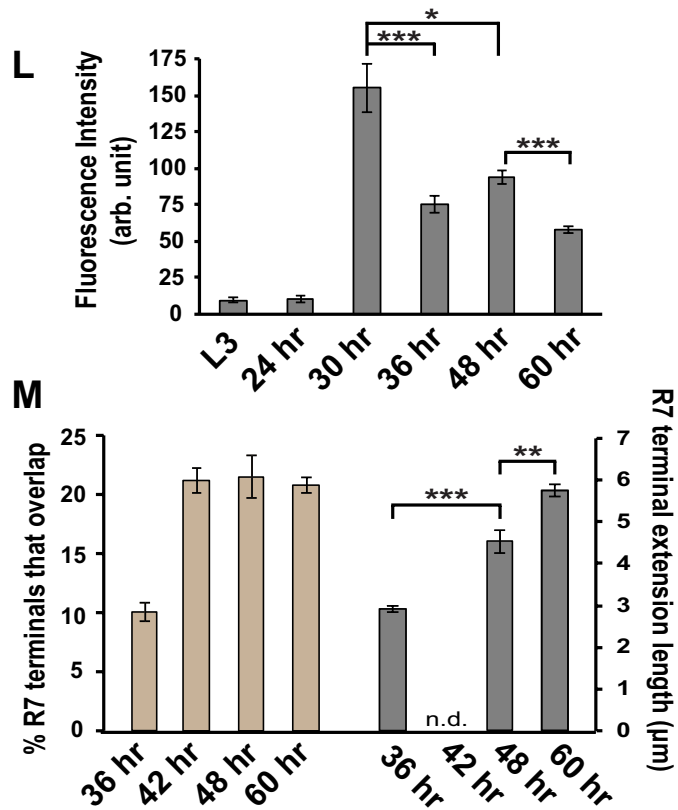

Figure 4. Ttk69 is normally first expressed in R7s after their axons extend into the medulla, and ttk69 mutant R7 axon overgrowth begins shortly afterward. $\boldsymbol{A}-\boldsymbol{E}^{\prime}$, Wild-type pupal retinas in which the R neurons are outlined by Phalloidin (red) and R7 nuclei express Pros (blue). Anti-Ttk69 is in green. $A, A^{\prime}$, At $24 \mathrm{~h} \mathrm{APF,} \mathrm{Ttk69} \mathrm{protein} \mathrm{is} \mathrm{absent} \mathrm{from} \mathrm{all} \mathrm{Rneurons} \mathrm{including} \mathrm{R7s} \mathrm{(arrow).} \boldsymbol{B}-\boldsymbol{E}^{\prime}$, From $30 \mathrm{~h}$ until at least

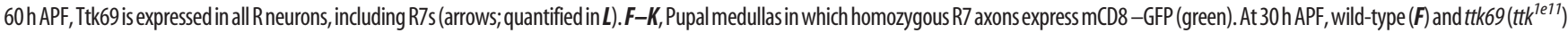
mutant (I) R7 axon terminals are indistinguishable. By $36 \mathrm{~h} \mathrm{APF,} \mathrm{ttk69} \mathrm{mutant} \mathrm{R7} \mathrm{axons} \mathrm{extend} \mathrm{laterally} \mathrm{within} \mathrm{the} \mathrm{M6} \mathrm{layer} \mathrm{(J).} \mathrm{By} 48 \mathrm{~h} \mathrm{APF,} \mathrm{ttk69} \mathrm{mutant} \mathrm{R7} \mathrm{axons} \mathrm{have} \mathrm{begun} \mathrm{to} \mathrm{extend} \mathrm{back} \mathrm{toward} \mathrm{M3} \mathrm{(K,}$ arrowhead). Quantified in M. L, Quantification of Ttk69 protein expression in wild-type R7 nuclei. Previous work has shown that Ttk69 protein is absent from third-instar larval R7s, suggesting that the $9.60 \pm$ $1.5(n=15)$ units of fluorescence we observe at that time point is caused by nonspecific background staining. At $24 \mathrm{~h} \mathrm{APF}$, Ttk69 protein remains absent from R7s $(9.97 \pm 2.3$ units; $n=11)$. However, by $30 \mathrm{~h}$ APF, R7s express a high level of Ttk69 (155 \pm 17 units; $n=10)$. This level drops somewhat by $36 \mathrm{~h} \mathrm{APF}(75.2 \pm 6.0$ units; $n=14)$ and further decreases by $60 \mathrm{~h}$ APF $(57.6 \pm 2.2$ units; $n=14)$. The differences between $30 \mathrm{~h}$ APF and the later time points are highly significant, as is the difference between 48 and $60 \mathrm{~h} \mathrm{APF}$. The difference between 36 and $48 \mathrm{~h}$ APF $(93.5 \pm 4.5$ units; $n=14)$ is not $(p=0.02) .{ }^{*} p<0.01$ and ${ }^{* * *} p<0.0001$. M, Quantification of $t$ tk69 $\left(t t^{\text {Te11 }}\right)$ mutant R7 axon extension over time. Error bars represent SEM. At $36 \mathrm{~h} \mathrm{APF,} 10.1 \pm 0.8 \%(n=10)$ of $t$ tk69 mutant R7 axons overlap with neighboring wild-type R7 axons. The frequency of ttk69 mutant R7 axon terminals that overlap with their neighbors peaks by $42 \mathrm{~h} \mathrm{APF}(21.2 \pm 1.1 \% ; n=11)$ and remains unchanged at $48 \mathrm{~h}$ APF $(21.6 \pm 1.8 \% ; n=10)$ and $60 \mathrm{~h} \mathrm{APF} \mathrm{(data} \mathrm{for} \mathrm{the} \mathrm{lattertime} \mathrm{point} \mathrm{is} \mathrm{redisplayed} \mathrm{from} \mathrm{Fig.} \mathrm{1Efor} \mathrm{ease} \mathrm{of} \mathrm{comparison).} \mathrm{The} \mathrm{average} \mathrm{length} \mathrm{of} \mathrm{the} \mathrm{ttk69R7} \mathrm{axon} \mathrm{terminal} \mathrm{extensions} \mathrm{continues} \mathrm{to} \mathrm{increase} \mathrm{even} \mathrm{after} \mathrm{the} \mathrm{frequency} \mathrm{of} \mathrm{overlap}$ has peaked: the difference between $36 \mathrm{~h} \mathrm{APF}(2.9 \pm 0.08 \mu \mathrm{m} ; n=10)$ and $48 \mathrm{~h} \mathrm{APF}(4.5 \pm 0.3 \mu \mathrm{m} ; n=10)$ is significant, as is the difference between 48 and $60 \mathrm{hAPF}(5.7 \pm 0.1 \mu \mathrm{m} ; n=9)$. ${ }^{* *} p<0.001$ and ${ }^{* * *} p<0.0001$. Scale bars, $5 \mu \mathrm{m}$. $n$ indicates number of brains or retinas scored.

filopodia-like processes toward their normal target layer (Fig. 5C). Even those Ttk69-expressing R7s that did reach the correct target layer had abnormally thin terminals. We observed similar results when we expressed Ttk69 in ttk69 mutant R7s (Fig. 5D). Because Ttk69, when expressed earlier, can transform R7 precursors into non-neuronal cells, we examined whether GMR-FLP/ MARCM-mediated Ttk69 expression altered R7 fate. We found that Ttk69-expressing R7s underwent their normal change in polarity and continued to express the photoreceptor-specific marker Chp (Fig. 5C), the neuron-specific marker Elav (data not shown), and the R7-specific marker Pros (Fig. 5F- $F^{\prime \prime}$ ), indicating that other aspects of R7 development were unaffected. In addi- tional support of the specificity of the effect of Ttk69, using GMR-FLP/MARCM to express the Ttk88 isoform had no effect on R7 growth (Fig. $5 E$ ), despite also being able to transform R7 precursors into non-neuronal cells if expressed earlier ( $\mathrm{Li}$ et al., 1997; Tang et al., 1997). Together, these results suggest that premature Ttk69 expression can collapse R7 growth cones and prevent them from advancing and that Ttk69 does so independently of its effect on neuronal differentiation.

Ttk69 is required for normal Activin signaling in R7s

Previous work has shown that the Activin receptor Babo is required to downregulate R7 axon growth during the second phase 
of targeting (Ting et al., 2007). We therefore wanted to test whether Ttk69 might act upstream or downstream of one or more components of this pathway. If Ttk69 controlled R7 axon growth independently of Activin signaling, we would predict that disrupting both Ttk69 and the Activin pathway would have a more severe effect on R7s than disrupting either alone. The most effective way to eliminate Activin signaling in R7s is to create large clones of babo mutant tissue using ey3.5FLP/MARCM (Ting et al., 2007). Consistent with previous reports, we found that $12.8 \pm 0.68 \%$ ( $n=9$ brains $)$ of babo mutant R7 axon terminals overlapped with their neighbors (Figs. 1D,6C). We found it technically easiest then to disrupt Ttk69 protein production in babo mutant clones by causing these clones to express a UAS$t t k-R N A i$ transgene. Expressing $t t k-R N A i$ in wild-type clones using ey3.5-FLP/ MARCM completely eliminated detectable Ttk69 in R7s (data not shown) and caused their axon terminals to overgrow at approximately the same frequency as ttk69 mutant R7 terminals (25.5 $\pm 0.66 \%$; $n=14$ brains; Fig. $6 A, C)$, suggesting that this method completely disrupts Ttk69 function. We found that the frequency of axon overgrowth was not increased by expressing $t t k-R N A i$ in babo mutant R7s (25.4 $\pm 1.3 \% ; n=12$ brain; Fig. $6 B, C)$, suggesting that Ttk69 and Babo likely function in the same pathway to regulate $\mathrm{R} 7$ axon growth.

We next wanted to test directly whether Ttk69 might act upstream or downstream of the Activin/Babo pathway. We found that Ttk69 is expressed at normal levels in babo mutant R7 nuclei during the second phase of R7 axon targeting (data not shown), suggesting that Ttk69 is not regulated by Babo. We therefore tested whether, instead, Activin signaling might be regulated by Ttk69. The output of Activin signaling can be assessed by measuring nuclear accumulation of the effector molecule dSmad2. A previous comparison of wild-type and babo mutant R7s expressing a UASFLAG-dSmad2 transgene showed that loss of babo significantly decreased the amount of FLAG-tagged dSmad2 present in R7 nuclei (Ting et al., 2007). We therefore tested whether loss of ttk69 might have a similar effect. We expressed UAS-FLAG-dSmad 2 in wild-type, $t$ tk69, and imp- $\alpha 3$ mutant R7s and quantified the amount of nuclear FLAG-tagged dSmad2 in each case (Fig. $\left.6 D, D^{\prime}\right)$. We found that loss of either $t$ tk 69 or imp- $\alpha 3$ significantly decreased nuclear dSmad2 compared with wild-type (Fig. 6E). We conclude that Ttk69 is required for normal Activin pathway signaling in R7s.

Ttk69 can also inhibit R7 axon growth independently of the Activin pathway

Although these results indicate that Ttk69 restricts R7 axon growth by promoting Activin pathway activity, disrupting the

\section{$24 \mathrm{hr}$ APF}

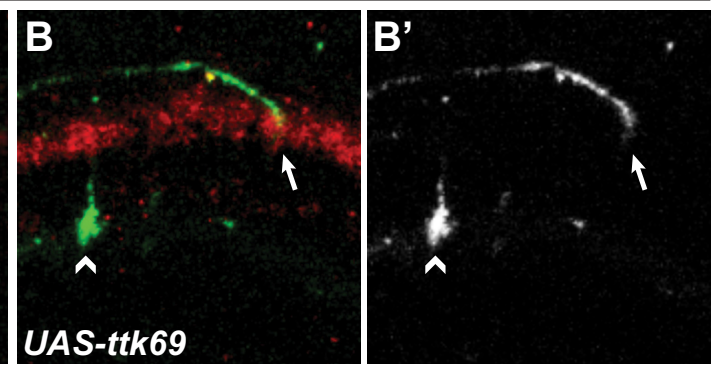

$48 \mathrm{hr}$ APF
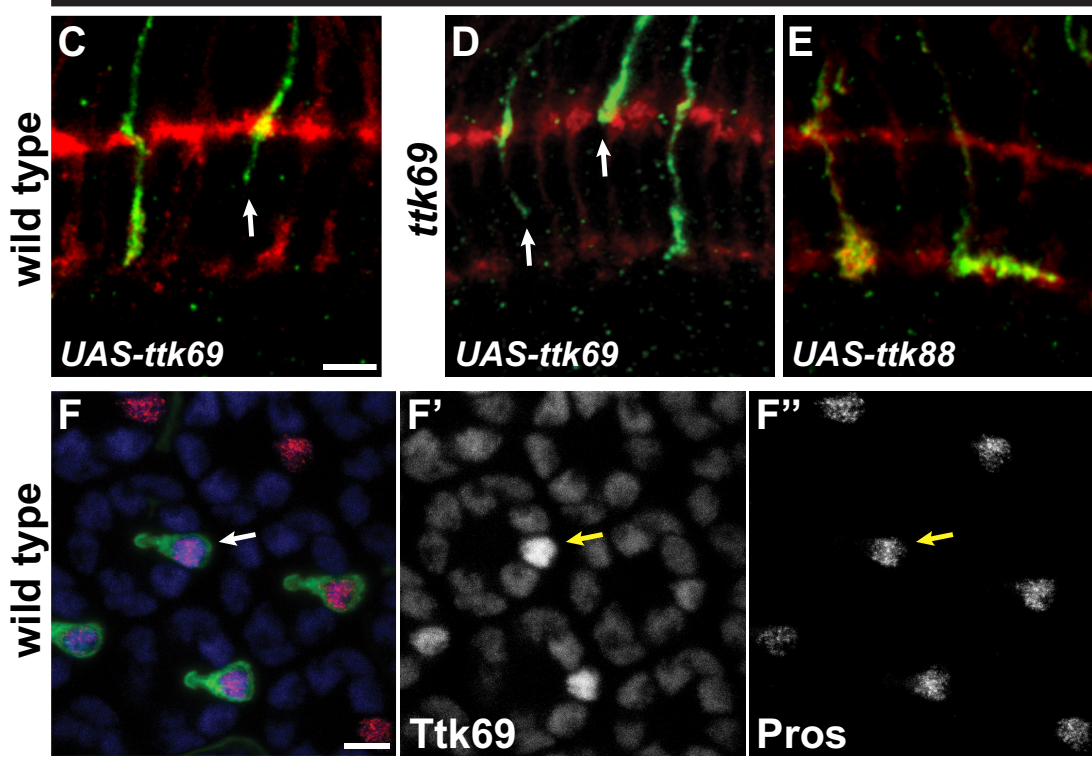

Figure 5. Premature Ttk69 expression disrupts R7 axon extension. $\boldsymbol{A}-\boldsymbol{E}$, Pupal medullas in which homozygous R7s, created by , wild-type $\mathrm{R} 7$ axons forced to express Ttk69 prematurely can have abnormally thin terminals (arrow), although some prematurely express Ttk69 fail to contact their target layer (arrow). D. Forced Ttk69 expression rescues the overgrowth of ttk 69 $\left(t t k^{1 e 11}\right)$ mutant R7 axons (see also Fig. $1 E$ ) but also prevents $14.3 \pm 1.6 \%(n=10)$ from contacting their target layer (arrows). $\boldsymbol{E}$, Forced Ttk88 expression neither rescues ttk69 mutant R7 axon overgrowth (see also Fig. 1E) nor prevents their axons from contacting their target layer. $\boldsymbol{F}-\boldsymbol{F}^{\prime \prime}$, At 48 h APF, the cell bodies of wild-type R7s that express Ttk69 prematurely (arrow) are normal in shape and express the R7 fate marker Pros. Scale bars, $5 \mu \mathrm{m}$. $n$ indicates number of brains scored.

Activin pathway in R7s does not cause as severe a defect as loss of Ttk69. babo mutant R7 axon terminals overlap with neighboring terminals less frequently $[12.8 \pm 0.68 \%, n=9$ (Fig. $6 C$ ) compared with $20.8 \pm 0.70 \%, n=9$ (Fig. $1 E$ ); $p<$ $0.00001]$ and have shorter extensions [2.6 $\pm 0.2 \mu \mathrm{m}(n=7)$ at $60 \mathrm{~h}$ APF compared with $5.7 \pm 0.1 \mu \mathrm{m}(n=9$; Fig. $4 M) ; p<$ $1 \times 10^{-8}$ ], and those babo mutant R7 terminals that do not overlap with their neighbors have terminals of normal size (Fig. 1D,F). This comparison suggests that Ttk69 might additionally inhibit R7 axon growth independently of the Activin pathway. To test this directly, we examined whether premature Ttk69 misexpression could inhibit R7 axon growth in the absence of Babo. We chose to disrupt Babo expression using a UAS-babo-RNAi transgene, because expressing Ttk69 in babo mutant clones using ey3.5-FLP/MARCM causes very early expression of Ttk69 and so blocks R7 differentiation (data not shown). We expressed both UAS-babo-RNAi and UAS-ttk69 

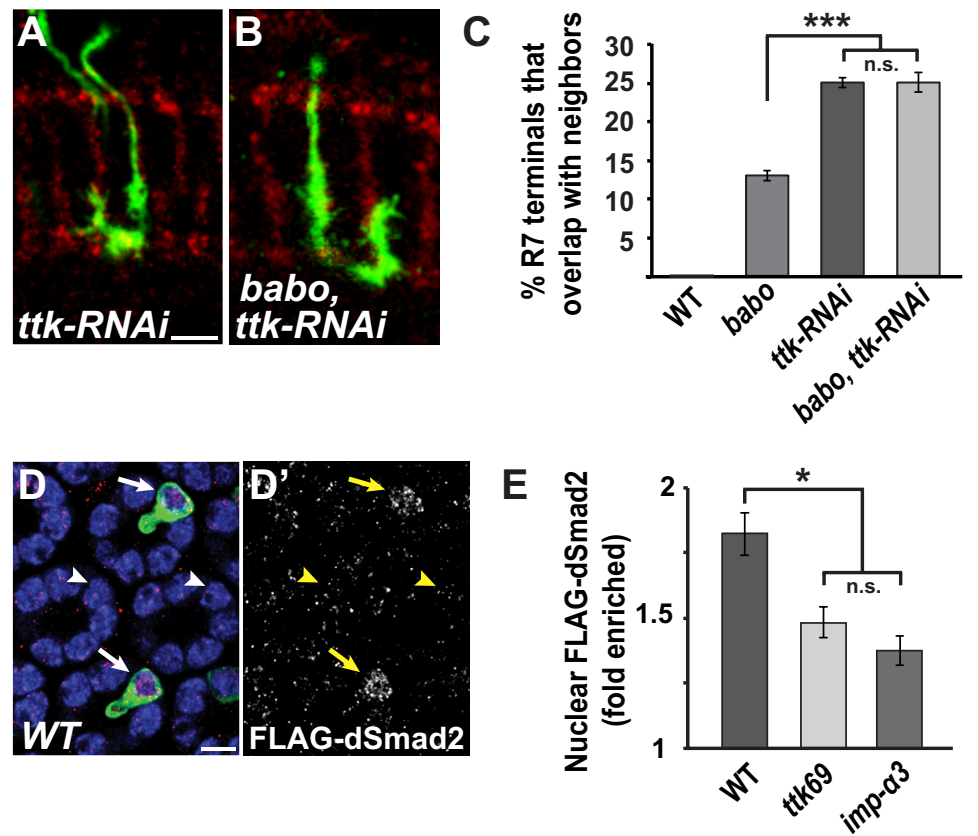

Figure 6. Ttk69 is required for normal Activin signaling in R7s. $A, B$, Medullas of mosaic animals, created by ey3.5-FLP/ MARCM, at $48 \mathrm{~h}$ APF. R7s homozygous for the specified chromosome arm express mCD8 -GFP (green). All R7 and R8 axons are labeled with anti-Chp (red). $\boldsymbol{A}$, Wild-type (FRT82) (WT) R7 axons that express ttk-RNAi extend laterally and overlap with neighboring R7 terminals at a frequency similar to that of $t$ tk69 mutant R7 axons. $B$, babo mutant R7 axons that express ttk-RNAi also extend laterally and overlap with neighboring R7 terminals. C, Quantification of R7 axon extension within M6. Error bars represent SEM. babo mutant R7 axons overlap with their neighbors significantly less often $(12.8 \% \pm$ $0.68 ; n=9)$ than wild-type R7 axons expressing ttk-RNAi $(25.5 \pm 0.66 ; n=14)$. However, removing babo from R7s that express $t t k-R N A i$ does not significantly increase the frequency of this defect $(25.4 \pm 1.3 \% ; n=12) . \boldsymbol{D}, \boldsymbol{D}^{\prime}, \mathrm{A} 50 \mathrm{~h}$ APF retina in which homozygous wild-type (FRT82) R7s (created by GMR-FLP/MARCM; arrows) express mCD8 -GFP (green) and FLAG-dSmad2 (red). All R neuron nuclei are labeled with anti-Elav (blue). Unlabeled R7 nuclei (arrowheads) do not express FLAG-dSmad2. $E$, Quantification of FLAG-dSmad2 levels in mutant R7 nuclei at $50 \mathrm{~h}$ APF. Error bars represent SEM. FLAG-dSmad2 staining in $t$ tk69 $\left(t t k^{1 e 11} ; 1.48 \pm 0.06 ; n=10\right)$ and $i m p-\alpha 3(1.37 \pm 0.06 ; n=10)$ mutant R7 nuclei is significantly reduced compared with that in wild-type R7 nuclei $(1.82 \pm 0.08 ; n=10)$. Scale bars, $5 \mu \mathrm{m}$. $n$ indicates number of brains or retinas scored. ${ }^{*} p<0.01$ and ${ }^{* * *} p<0.0001$

using chp-Gal4, which initiates expression in $\mathrm{R}$ neurons after their specification as neurons (data not shown). We found that expressing $b a b o-R N A i$ alone caused $\mathrm{R} 7$ axon terminals to overlap with their neighbors at the same frequency as babo mutant R7 terminals $(13.9 \pm 0.9 \% ; n=8$; Fig. $7 A, D)$. Expressing Ttk69 alone using chp-Gal4 caused R7 axons to stop short of their final target layer only slightly less often than GMR-FLP/MARCM/ UAS-ttk69 R7 axons $[11.5 \pm 0.6 \%, n=11$ (Fig. $7 B, D$ ) compared with $17.6 \pm 1.3 \%, n=9$ (Fig. $5 C$ ) ] and did not disrupt their neuronal identity (data not shown). We therefore examined the effects of expressing Ttk69 and babo-RNAi simultaneously. We found that forced Ttk69 expression caused only a mild reduction in the frequency with which Babo-lacking R7 axons extended laterally within M6 (Fig. 7C,D), suggesting that Ttk69 acts upstream of Babo to promote Activin signaling. However, if this were the only mechanism by which Ttk69 restricts R7 axon growth, then disrupting Babo should similarly prevent Ttk69 from causing premature arrest of R7 axons. Instead, disrupting Babo did not significantly decrease the frequency with which Ttk69-expressing R7s failed to reach M6 (Fig. 7D). Together with the observed difference between the ttk69 and babo loss-offunction phenotypes, these results suggest that Ttk69 can inhibit R7 axon growth independently of the Activin pathway.

\section{Discussion}

Ttk69 inhibits axon growth during the second phase of R7 axon targeting We have shown that Ttk69 is both necessary and sufficient to prevent R7 axons from extending. Because $t$ tk69 mutant R7s remain responsive to repulsion by their neighbors and can initiate synapse assembly with the appropriate target layers, we hypothesize that Ttk69 regulates the intrinsic ability of R7 axon terminals to grow. Consistent with this possibility, Ttk69 regulates the morphology of R7 growth cones: loss of Ttk69 increases R7 growth cone size, whereas premature Ttk69 causes R7 growth cone collapse. We have found that Ttk69 is expressed and required specifically during the second phase of R7 axon targeting, after the R7 axons have reached the medulla. A simple model is that R7 axon terminals are normally stationary throughout this second phase, even as the layer they occupy slowly advances, and that Ttk69 is deployed to establish and maintain this stationary state. In support of this model, we note that $t$ tk69 mutant R7 growth cones begin extending laterally quite early in the second phase of targeting, long before they have reached M6. However, the laterally extended sections of $t$ tk 69 mutant R7 axons always remain in the same layer as the neighboring wild-type R7 axon terminals, even as the length of the lateral extensions continues to increase. One possibility is that the entire lateral length of each $t t k 69$ mutant R7 axon remains motile and extends in step with wild-type R7 growth cones. However, we think it more likely that, as the optic lobe expands, the temporary target layer to which the R7 axon terminals adhere is passively driven forward by the intercalation of additional neuronal processes, causing the R7 axons to elongate by "stretch growth" (Smith, 2009) rather than by growth cone extension. Assuming that Ttk69 does not affect the ability of R7 axons to adhere to their target layer, then the full lateral length of the $t$ tk69 mutant R7 axons would be pulled forward together with adjacent wild-type R7 terminals. In support of this model, we found that $t$ tk 69 mutant R7 axons can localize synaptic vesicles normally, indicating that loss of Ttk69 does not prevent R7 axons from interacting with their targets.

\section{Ttk69 expression in R7s must be temporally regulated}

Premature Ttk69 expression prevents R7 axons from reaching their targets, and $t t k 69$ mutant $\mathrm{R} 7$ axon terminals begin to overgrow soon after the time at which Ttk69 would normally be expressed. We conclude that the temporal regulation of Ttk69 expression is critical for normal R7 axon targeting. How might this regulation be achieved? During larval development, R7s eliminate Ttk69 by initiating expression of the adaptor protein Phyl, which targets Ttk69 for ubiquitination by the RING finger protein Sina and subsequent degradation by the proteosome ( $\mathrm{Li}$ et al., 1997; Li et al., 2002). We found that expressing Phyl in pupal R7s was sufficient to eliminate Ttk69 expression and 
caused axon overgrowth (data not shown), suggesting that pupal R7s continue to express Sina. However, attempting to disrupt pupal expression of Phyl or Sina (by delayed expression of the corresponding UAS-RNAi transgenes using GMR-FLP/MARCM/UASRNAi) did not cause premature Ttk69 expression or R7 growth cone collapse (data not shown), perhaps because of partial redundancy with the translational repressor Musashi (Hirota et al., 1999). We therefore do not yet know whether R7s regulate both larval and pupal Ttk69 expression by the same mechanism. However, it is clear that R7s do not initiate Ttk69 expression simply based on their age: although there is at least a $38 \mathrm{~h}$ range of $\mathrm{R} 7$ ages in the pupal eye (Wolff and Ready, 1993), we found that all R7s express Ttk69 within a $6 \mathrm{~h}$ window. One possibility is that Ttk69 expression is initiated simultaneously in R7s by a signal from the optic lobe-a signal that would then require retrograde axonal transport for transduction to the nucleus. Although disrupting axonal transport by expressing a dominantnegative form of the Dynactin subunit Glued $\left(\mathrm{Gl}^{\mathrm{DN}}\right)$ causes R7 axon to extend laterally within M6 (Ting et al., 2007), we found that R7s expressing $\mathrm{Gl}^{\mathrm{DN}}$ had normal levels of nuclear Ttk69 (as judged at $48 \mathrm{~h}$ APF; data not shown). We conclude that Ttk69 expression in R7s is likely to be independent of target- or growth-cone-derived signals. Instead, R7 Ttk69 expression may be controlled hormonally or by signaling within the retina, or R7s may use a cell-intrinsic mechanism that does not rely solely on R7 age.

\section{Ttk69 promotes Activin pathway activity in $\mathrm{R} 7 \mathrm{~s}$}

Our results indicate that Ttk69 restricts R7 axon growth in part by promoting Activin pathway activity. Because Ttk69 is a zinc finger transcription factor and because we find that nuclear localization of Ttk69 is required to prevent R7 axon overgrowth, the simplest model is that Ttk69 regulates the transcription of one or more Activin pathway components. Our finding that Ttk69 overexpression does not rescue Babo loss suggests that Ttk69 acts upstream of Babo. Because previous work indicated that the Activin signal may originate from the R7s themselves (Ting et al., 2007), a simple possibility is that Ttk69 promotes Activin expression. However, because loss of Ttk69 reduces but does not completely disrupt Activin pathway activity and because Ttk69 is a well-established repressor (Xiong and Montell, 1993; Reddy et al., 2010), we speculate that Ttk69 might instead repress an antagonist of the Activin ligand. We note that the locus encoding one such antagonist, Follistatin, contains a potential Ttk69binding DNA sequence (Harrison et al., 2005; data not shown).

Canonical Activin signaling in Drosophila is transduced to the nucleus by the transcription factor dSmad2 (Brummel et al., 1999; Das et al., 1999; Zheng et al., 2003). Previous work found that loss of dSmad 2 caused considerably less R7 axon overgrowth than loss of Babo (Ting et al., 2007), suggesting that Babo may additionally regulate $\mathrm{R} 7$ axon outgrowth by a Smad-independent mechanism. Although overexpressing regulatory Smads can compensate for reduced canonical TGF- $\beta$ pathway activation, we note that overexpressing FLAG-dSmad2 did not decrease the R7 axon overgrowth caused by loss of ttk69 (data not shown). We therefore speculate that dSmad2 may not be the only output of Activin signaling in R7 neurons and that, as in Drosophila mushroom body (Ng, 2008), Activin - and therefore Ttk69may also inhibit R7 axon outgrowth by a non-canonical, Smad-independent mechanism.

\section{Ttk69 regulates the morphology of multiple cell types}

Ttk69 is broadly expressed in non-neuronal cells and is both necessary and sufficient to block the adoption of neural fates (Read and Manley, 1992; Guo et al., 1995; Giesen et al., 1997; Badenhorst, 2001; Badenhorst et al., 2002). Although cells destined to become neurons must therefore eliminate Ttk69 expression, we have here shown that $\mathrm{R} 7$ neurons later reexpress Ttk69 to inhibit axon growth. Might other neurons use Ttk69 to regulate neurite outgrowth? We note that the R1-R6 and R8 photoreceptor neurons also reexpress Ttk69 after their axons have reached temporary target layers in the optic lobe (Fig. 4), suggesting that Ttk69 may play such a role in these cells. In addition, loss or gain of $t$ tk disrupts the morphology of dendrite arborization neurons in the peripheral nervous system (Parrish et al., 2006; Ou et al., 2008), suggesting that 
Ttk69 may regulate dendrite growth. Recent work has shown that Ttk69 also regulates the morphology of several non-neuronal cell types. Loss of Ttk69 from a subset of follicle cells prevents them from undergoing a cell shape change required for proper elongation of the dorsal appendage (French et al., 2003; Boyle and Berg, 2009; Boyle et al., 2010), and loss of Ttk69 from tracheal cells disrupts their ability to elongate during intercalation (Araújo et al., 2007). Together, these results suggest that Ttk69 may play a general role in temporally coordinating pathways that regulate cytoskeleton in both neuronal and non-neuronal cells.

\section{References}

Allen MJ, Shan X, Caruccio P, Froggett SJ, Moffat KG, Murphey RK (1999) Targeted expression of truncated glued disrupts giant fiber synapse formation in Drosophila. J Neurosci 19:9374-9384. Medline

Araújo SJ, Cela C, Llimargas M (2007) Tramtrack regulates different morphogenetic events during Drosophila tracheal development. Development 134:3665-3676. CrossRef Medline

Ashley JA, Katz FN (1994) Competition and position-dependent targeting in the development of the Drosophila R7 visual projections. Development 120:1537-1547. Medline

Astigarraga S, Hofmeyer K, Treisman JE (2010) Missed connections: photoreceptor axon seeks target neuron for synaptogenesis. Curr Opin Genet Dev 20:400-407. CrossRef Medline

Badenhorst P (2001) Tramtrack controls glial number and identity in the Drosophila embryonic CNS. Development 128:4093-4101. Medline

Badenhorst P, Finch JT, Travers AA (2002) Tramtrack co-operates to prevent inappropriate neural development Drosophila. Mech Dev 117:87-101. CrossRef Medline

Boyle MJ, Berg CA (2009) Control in time and space: Tramtrack69 cooperates with Notch and Ecdysone to repress ectopic fate and shape changes during Drosophila egg chamber maturation. Development 136:4187-4197. CrossRef Medline

Boyle MJ, French RL, Cosand KA, Dorman JB, Kiehart DP, Berg CA (2010) Division of labor: subsets of dorsal-appendage-forming cells control the shape of the entire tube. Dev Biol 346:68 -79. CrossRef Medline

Brummel T, Abdollah S, Haerry TE, Shimell MJ, Merriam J, Raftery L, Wrana JL, O'Connor MB (1999) The Drosophila activin receptor baboon signals through $\mathrm{dSmad} 2$ and controls cell proliferation but not patterning during larval development. Genes Dev 13:98-111. CrossRef Medline

Choe KM, Prakash S, Bright A, Clandinin TR (2006) Liprin-alpha is required for photoreceptor target selection in Drosophila. Proc Natl Acad Sci U S A 103:11601-11606. CrossRef Medline

Clandinin TR, Lee CH, Herman T, Lee RC, Yang AY, Ovasapyan S, Zipursky SL (2001) Drosophila LAR regulates R1-R6 and R7 target specificity in the visual system. Neuron 32:237-248. CrossRef Medline

Das P, Inoue H, Baker JC, Beppu H, Kawabata M, Harland RM, Miyazono K, Padgett RW (1999) Drosophila dSmad2 and Atr-I transmit activin/TGFbeta signals. Genes Cells 4:123-134. CrossRef Medline

Dietzl G, Chen D, Schnorrer F, Su KC, Barinova Y, Fellner M, Gasser B, Kinsey K, Oppel S, Scheiblauer S, Couto A, Marra V, Keleman K, Dickson BJ (2007) A genome-wide transgenic RNAi library for conditional gene inactivation in Drosophila. Nature 448:151-156. CrossRef Medline

French RL, Cosand KA, Berg CA (2003) The Drosophila female sterile mutation twin peaks is a novel allele of tramtrack and reveals a requirement for Ttk69 in epithelial morphogenesis. Dev Biol 253:18-35. CrossRef Medline

Giesen K, Hummel T, Stollewerk A, Harrison S, Travers A, Klämbt C (1997) Glial development in the Drosophila CNS requires concomitant activation of glial and repression of neuronal differentiation genes. Development 124:2307-2316. Medline

Guo M, Bier E, Jan LY, Jan YN (1995) tramtrack acts downstream of numb to specify distinct daughter cell fates during asymmetric cell divisions in the Drosophila PNS. Neuron 14:913-925. CrossRef Medline

Hadjieconomou D, Timofeev K, Salecker I (2011) A step-by-step guide to visual circuit assembly in Drosophila. Curr Opin Neurobiol 21:7684. CrossRef Medline

Harrison CA, Gray PC, Vale WW, Robertson DM (2005) Antagonists of activin signaling: mechanisms and potential biological applications. Trends Endocrinol Metab 16:73-78. CrossRef Medline

Hirota Y, Okabe M, Imai T, Kurusu M, Yamamoto A, Miyao S, Nakamura M,
Sawamoto K, Okano H (1999) Musashi and Seven in absentia downregulate Tramtrack through distinct mechanisms in Drosophila eye development. Mech Dev 87:93-101. CrossRef Medline

Hofmeyer K, Maurel-Zaffran C, Sink H, Treisman JE (2006) Liprin- $\alpha$ has LAR-independent functions in R7 photoreceptor axon targeting. Proc Natl Acad Sci U S A 103:11595-11600. CrossRef Medline

Holbrook S, Finley JK, Lyons EL, Herman TG (2012) Loss of syd-1 from R7 neurons disrupts two distinct phases of presynaptic development. J Neurosci 32:18101-18111. CrossRef

Kauffmann RC, Li S, Gallagher PA, Zhang J, Carthew RW (1996) Ras1 signaling and transcriptional competence in the R7 cell of Drosophila. Genes Dev 10:2167-2178. CrossRef Medline

Kolodkin AL, Tessier-Lavigne M (2011) Mechanisms and molecules of neuronal wiring: a primer. Cold Spring Harv Perspect Biol 3:a001727. CrossRef Medline

Lai ZC, Li Y (1999) Tramtrack69 is positively and autonomously required for Drosophila photoreceptor development. Genetics 152:299-305. Medline

Lee CH, Herman T, Clandinin TR, Lee R, Zipursky SL (2001) N-cadherin regulates target specificity in the Drosophila visual system. Neuron 30: 437-450. CrossRef Medline

Lee T, Luo L (1999) Mosaic analysis with a repressible cell marker for studies of gene function in neuronal morphogenesis. Neuron 22:451-461. CrossRef Medline

Li S, Li Y, Carthew RW, Lai ZC (1997) Photoreceptor cell differentiation requires regulated proteolysis of the transcriptional repressor Tramtrack. Cell 90:469-478. CrossRef Medline

Li S, Xu C, Carthew RW (2002) Phyllopod acts as an adaptor protein to link the sina ubiquitin ligase to the substrate protein tramtrack. Mol Cell Biol 22:6854-6865. CrossRef Medline

Mason DA, Máthé E, Fleming RJ, Goldfarb DS (2003) The Drosophila melanogaster importin alpha3 locus encodes an essential gene required for the development of both larval and adult tissues. Genetics 165 : 1943-1958. Medline

Maurel-Zaffran C, Suzuki T, Gahmon G, Treisman JE, Dickson BJ (2001) Cell-autonomous and -nonautonomous functions of LAR in R7 photoreceptor axon targeting. Neuron 32:225-235. CrossRef Medline

McQuilton P, St Pierre SE, Thurmond J, Flybase Consortium (2012) Flybase 101-the basics of navigating FlyBase. Nucleic Acids Res 40 [Database issue]:D706-D714. CrossRef

Miller AC, Lyons EL, Herman TG (2009) cis-Inhibition of Notch by endogenous Delta biases the outcome of lateral inhibition. Curr Biol 19:1378 1383. CrossRef Medline

Moore DL, Goldberg JL (2011) Multiple transcription factor families regulate axon growth and regeneration. Dev Neurobiol 71:1186-1211. CrossRef Medline

Mullins MC, Rubin GM (1991) Isolation of temperature-sensitive mutations of the tyrosine kinase receptor sevenless (sev) in Drosophila and their use in determining its time of action. Proc Natl Acad Sci U S A 88:9387-9391. CrossRef Medline

Ng J (2008) TGF-beta signals regulate axonal development through distinct Smad-independent mechanisms. Development 135:4025-4035. CrossRef Medline

Ou Y, Chwalla B, Landgraf M, van Meyel DJ (2008) Identification of genes influencing dendrite morphogenesis in developing peripheral sensory and central motor neurons. Neural Dev 3:16. CrossRef Medline

Parrish JZ, Kim MD, Jan LY, Jan YN (2006) Genome-wide analyses identify transcription factors required for proper morphogenesis of Drosophila sensory neuron dendrites. Genes Dev 20:820-835. CrossRef Medline

Read D, Manley JL (1992) Alternatively spliced transcripts of the Drosophila tramtrack gene encode zinc finger proteins with distinct DNA binding specificities. EMBO J 11:1035-1044. Medline

Reddy BA, Bajpe PK, Bassett A, Moshkin YM, Kozhevnikova E, Bezstarosti K, Demmers JA, Travers AA, Verrijzer CP (2010) Drosophila transcription factor Tramtrack69 binds MEP1 to recruit chromatin remodeler NuRD. Mol Cell Biol 30:5234-5244. CrossRef Medline

Schindelin J, Arganda-Carreras I, Frise E, Kaynig V, Longair M, Pietzsch T, Preibisch S, Rueden C, Saalfeld S, Schmid B, Tinevez JY, White DJ, Hartenstein V, Eliceiri K, Tomancak P, Cardona A (2012) Fiji: an opensource platform for biological-image analysis. Nat Methods 9:676-682. CrossRef Medline

Siddall NA, Hime GR, Pollock JA, Batterham P (2009) Ttk69-dependent 
repression of lozenge prevents the ectopic development of R7 cells in the Drosophila larval eye disc. BMC Dev Biol 9:64. CrossRef Medline

Smith DH (2009) Stretch growth of integrated axon tracts: extremes and exploitations. Prog Neurobiol 89:231-239. CrossRef Medline

Sun F, He Z (2010) Neuronal intrinsic barriers for axon regeneration in the adult CNS. Curr Opin Neurobiol 20:510-518. CrossRef Medline

Takemura SY, Lu Z, Meinertzhagen IA (2008) Synaptic circuits of the Drosophila optic lobe: the input terminals to the medulla. J Comp Neurol 509:493-513. CrossRef Medline

Tang AH, Neufeld TP, Kwan E, Rubin GM (1997) PHYL acts to downregulate TTK88, a transcriptional repressor of neuronal cell fates, by a SINAdependent mechanism. Cell 90:459-467. CrossRef Medline

Ting CY, Yonekura S, Chung P, Hsu SN, Robertson HM, Chiba A, Lee CH (2005) Drosophila N-cadherin functions in the first stage of the two-stage layer-selection process of R7 photoreceptor afferents. Development 132: 953-963. CrossRef Medline

Ting CY, Herman T, Yonekura S, Gao S, Wang J, Serpe M, O'Connor MB,
Zipursky SL, Lee CH (2007) Tiling of R7 axons in the Drosophila visual system is mediated both by transduction of an Activin signal to the nucleus and by mutual repulsion. Neuron 56:793-806. CrossRef Medline

Vitriol EA, Zheng JQ (2012) Growth cone travel in space and time: the cellular ensemble of cytoskeleton, adhesion, and membrane. Neuron 73: 1068-1081. CrossRef Medline

Wolff T, Ready DF (1993) Pattern formation in the Drosophila retina. In: The development of Drosophila melanogaster (Bate M, Arias AM eds), pp 1277-1325. Cold Spring Harbor, NY: Cold Spring Harbor Laboratory.

Xiong WC, Montell C (1993) tramtrack is a transcriptional repressor required for cell fate determination in the Drosophila eye. Genes Dev 7:1085-1096. CrossRef Medline

Zheng X, Wang J, Haerry TE, Wu AY, Martin J, O'Connor MB, Lee CH, Lee T (2003) TGF-beta signaling activates steroid hormone receptor expression during neuronal remodeling in the Drosophila brain. Cell 112:303-315. CrossRef Medline 